\title{
Mercury in fishes from Augusta Bay (southern Italy): Risk assessment and health implication
}

\author{
M. Bonsignore ${ }^{\text {a }}$, D. Salvagio Manta ${ }^{\text {b }}$, E. Oliveri ${ }^{\text {a }}$, M. Sprovieri ${ }^{\text {a,* }}$, G. Basilone ${ }^{\text {a }}$, A. Bonanno ${ }^{\text {a }}$, F. Falco $^{\text {a }}$, \\ A. Traina ${ }^{\text {a }}$ S. Mazzola ${ }^{a}$ \\ a Institute for Coastal and Marine Environment (IAMC) - CNR, Via del Mare, 3, 91021 Torretta Granitola, Campobello di Mazara (TP), Italy \\ ${ }^{\mathrm{b}}$ Institute for Coastal and Marine Environment (IAMC) - CNR, Calata Porta di Massa, 80100 Naples, Italy
}

\section{A R T I C L E I N F O}

\section{Article history:}

Received 15 December 2012

Accepted 14 February 2013

Available online 24 February 2013

\section{Keywords:}

Mercury

Fishes

Pollution effect

Bioaccumulation

Toxicity

\begin{abstract}
A B S T R A C T
Our study reports on the total mercury (HgT) concentrations measured in the muscles and livers of several benthic, demersal and pelagic fish species caught inside and outside of Augusta Bay (southern Italy), a semi-enclosed marine area, highly contaminated by the uncontrolled (since the 1950s to 1978s) discharge of the largest European petrochemical plant. Mercury levels in fish tissues are discussed with regard to specific habitat, size and/or age of the specimens and HgT distribution in the bottom sediments. Results suggest a still active $\mathrm{Hg}$ release mechanism from the polluted sediments to the marine environment. Also, the high HgT concentrations measured in fishes caught in the external area of the bay imply a potential role of Augusta Bay as a pollutant source for the Mediterranean ecosystem. Finally, values of hazard target quotient (THQ) and estimated weekly intake (EWI) demonstrate that consumption of fishes caught inside the bay represents a serious risk for human health. Also, data indicate that intake of fishes caught from the external area of the bay, especially for that concern demersal and benthic species, could be represent a significant component of risk for the local population.
\end{abstract}

(c) 2013 Elsevier Ltd. All rights reserved.

\section{Introduction}

The city of Augusta, located in the SE of Sicily (southern Italy), has experienced an important industrialisation phase since the early 60s. This has led to the creation of several chemical and petrochemical plants and oil refineries resulting in a severe pollution of the surrounding environment. In particular, the petrochemical industry in Augusta Bay is one of the largest in Europe with the most important chlor-alkali plant in Italy (Le Donne and Ciafani, 2008). Its activity started in 1958 and stopped in 2005, with production of chlorine and caustic soda by electrolysis of sodium chloride aqueous solution in electrolytic cells with a graphite anode and metallic mercury cathode. Uncontrolled chemical discharge of $\mathrm{Hg}$ occurred in the Augusta Bay until 1978, when restrictions were imposed by the Italian legislation.

In the last decade, several studies have provided detailed information on the pollution levels and risks for human health of resident populations of Augusta Bay (ICRAM, 2005; Ausili et al., 2008; Di Leonardo et al., 2007, 2008; ENVIRON International Team, 2008; Ficco et al., 2009; Sprovieri et al., 2011). Sprovieri et al. (2011) reported high-resolution maps of HgT distribution from superficial sediments collected in 2005 , highlighting extremely

\footnotetext{
* Corresponding author. Tel.: +39 092440670; fax: +39 092440445.

E-mail address: mario.sprovieri@iamc.cnr.it (M. Sprovieri).
}

high concentrations (ranging between 0.1 and $527.3 \mathrm{mg} \mathrm{kg}^{-1}$ ) and speculating on the key role that Augusta Bay could play in exporting $\mathrm{Hg}$ to the Mediterranean Sea, as an effect of the outflow intercepted by the Levantine Intermediate Waters (LIWs). Also, data recently collected by ICRAM (2008), ENVIRON International Team (2008) and Ausili et al. (2008), demonstrated HgT transfer from the abiotic system (sediments and seawater) to fishes (top predators and filter-feeders) and documented significant health risks associated with the consumption of fish caught in the area. Toxicological $\mathrm{Hg}$ effects were also evaluated on mussels and red mullet by micronuclei (MN) studies, which documented DNA damage (Ausili et al., 2008 and ICRAM, 2008). Finally, Tomasello et al. (2012) report on DNA genotoxic and oxidative damages in Coris julis specimens from Augusta Bay.

Fish food seems to constitute the main route of $\mathrm{Hg}$ uptake for humans (Holsbeek et al., 1996; Nakagawa et al., 1997).

Renzoni et al. (1998) demonstrated that long-term and frequent intake of fish with high $\mathrm{Hg}$ levels is statistically associated with a toxic risk, especially in pregnant women. A sad, famous poisoning episode occurred in the 1950s among people living around Minamata Bay (Japan), showing the irreversible neurological damage and teratogenic effects due to consummation of $\mathrm{Hg}$-contaminated fish (De Flora et al., 1994). Methylmercury ( $\mathrm{MeHg}$ ) is the most toxic form, able to interfere with thiol metabolism, causing inhibition or inactivation of proteins containing thiol ligands and ultimately 
leading to mitotic disturbances (Das et al., 1982; Elhassani, 1983). Numerous recent studies indeed have concluded that the majority, if not all, of the $\mathrm{Hg}$ that is bioaccumulated through the food chain is as MeHg (Winfrey and Rudd, 1990; Mason and Fitzgerald, 1990, 1991; Gilmour and Henry, 1991; Horvat et al., 1999; Carbonell et al., 2009).

High mortality rates, statistical high frequency of neonatal malformations and cancerous diseases reported for resident populations around Augusta Bay (Martuzzi et al., 2006; Bianchi et al., 2004, 2006; Fano et al., 2005, 2006; Madeddu et al., 2006) definitively calls for more detailed exploration and definitive assessment of the role played by the intake of $\mathrm{Hg}$-contaminated fish on the health of the consumers.

In this work, we aim to explore the effects of HgT pollution in Augusta Bay on the fish compartment, inside and outside the semi-enclosed area, and to assess the potential health risks associated with the consumption of contaminated fish.

\section{Materials and methods}

\subsection{Sampling}

Four different sampling sites were selected: two inside, and two outside of Augusta Bay (Fig. 1). Sampling outside the bay was performed during May 2001, on board of the N/O "Dallaporta", by means of a mid-water trawl-net at 50-100 m of depth in two sampling areas, in front of the Scirocco inlet (300-m wide and 13-m deep), and the Levante inlet (400-m wide and 40-m deep) (Fig. 1: C1, C2). Mainly pelagic fish specimens were caught (Table 1). Sampling inside the bay was performed during May 2012 by means of a fishing boat equipped with a gillnet wall, positioned at the bottom (mean depth $=20-25 \mathrm{~m}$ ) (Fig. 1: C3, C4). Several specimens of benthic and demersal fishes were collected. From the two sampling activities, a total of 227 fish specimens were collected: 107 from mid-water sampling (outside the bay) and 120 from bottom-water sampling (inside the bay). Moreover specimens of Engraulis encrasicolus $(n=38)$ were caught from the unpolluted marine area of Marsala (western Sicily) (Fig. 1), during July 2001, on board of a fishing boat equipped with a purse seine net. After collection, fishes were stored at $-20^{\circ} \mathrm{C}$ until biological and chemical analyses were performed in the laboratories of biology and biogeochemistry at the Institute for Coastal and Marine Environment (CNR) of Capo Granitola.

\subsection{Biological data and tissue collection}

The total length (TL) of each specimen was measured. Muscle and liver tissues were collected from each organism, using plastic materials cleaned with $\mathrm{HNO}_{3}$ (10\%) and MilliQ water, in order to avoid $\mathrm{Hg}$ contamination. Tissues were stored at $-20^{\circ} \mathrm{C}$ until analysis. Otoliths were extracted from anchovy and sardine specimens for age determination. Readings and interpretation of otolith increment growths were carried out by transmitted visible lights based on higher-resolution microscopy (20-25× magnification) (Campana et al., 1987; Nielsen, 1992). The procedure adopted for European anchovy age determination follow Uriarte et al. (2007) and La Mesa et al. (2009).

\subsection{Chemical analyses}

Total mercury concentrations ( $\mathrm{HgT}$ ) in tissues were measured using a direct mercury analyser (Milestone_DMA-80), atomic absorption spectrophotometer, according to analytical procedures reported in EPA 7473. Briefly, approximately $0.1 \mathrm{~g}$ of fresh tissue was loaded in nickel boats and transferred to the DMA-80 system. In order to minimise contamination risks, acid-cleaned laboratory materials were used during sample preparation and analyses. A Reference Standard Material (TORT-2; HgT certificate value $=0.27 \pm 0.06 \mu \mathrm{g} \mathrm{g}^{-1}$ ) was analysed to assess analytical accuracy (estimated to be $\sim 3 \%$ ) and precision (routinely better than $4 \%$; RSD\%, $n=3$ ). Finally, duplicated samples (about $20 \%$ of the total number of samples) were measured to estimate reproducibility, which resulted in better than $7 \%$.

\subsection{THQ and EWI calculation}

Target hazard quotient (THQ) and estimated weekly intake (EWI) were calculated for muscles of fishes caught inside and outside the bay.

The target hazard quotient was calculated according to the US EPA (1989) method and it is described by the following equation:

$\mathrm{THQ}=\left(\frac{\mathrm{EF} \times \mathrm{ED} \times \mathrm{FIR} \times C}{\mathrm{RFD} \times \mathrm{WAB} \times \mathrm{TA}}\right) \times 10^{-3}$ where EF is exposure frequency (365 days/year); ED is the exposure duration (70 years), equivalent to the average lifetime; FIR is the food ingestion rate ( $36 \mathrm{~g} / \mathrm{per}$ son/day) (FAO, 2005); $C$ is the metal concentration in seafood $\left(\mu \mathrm{g} \mathrm{g}^{-1}\right)$; RFD is the USEPA's reference dose $\left(0.1 \mu \mathrm{g} \mathrm{Hg} \mathrm{kg} \mathrm{bw}{ }^{-1} \mathrm{~d}^{-1}\right)$ (http://cfpub.epa.gov) or acceptable daily intake determined by WHO $\left(0.23 \mu \mathrm{g} \mathrm{Hg} \mathrm{kg} \mathrm{bw}^{-1} \mathrm{~d}^{-1}\right)$ (http://apps.who.int); WAB is the average body weight $(60 \mathrm{~kg})$, and TA is the average exposure time for no carcinogens ( 365 days/year $\times$ ED).

The THQ was calculated for all the studied species in the Augusta Bay using the US-EPA's reference dose (THQa) and the acceptable daily intake determined by the $\mathrm{WHO}(\mathrm{THQb})$. In particular, we assumed that the measured mercury is integrally in its methylated form (Winfrey and Rudd, 1990; Mason and Fitzgerald, 1990, 1991; Gilmour and Henry, 1991; Horvat et al., 1999; Carbonell et al., 2009).

The estimated weekly intake (EWI) was calculated by multiplying the HgT concentration (C) times by the weekly dietary intakes (FIR $\times 7$ ) and reporting to the average body weight (WAB).

Finally, mean THQ and EWI values were calculated for each studied species. Also, considering that fishing activity within the bay has been interdicted since 2007 (Order No. 73/07), data relative to fishes from inside and outside the bay were processed separately.

\section{Results}

\subsection{Biological features}

The fish caught from bottom-water sampling (inside the bay) consisted of 2 pelagic, 106 demersal and 16 benthic specimens, while specimens from mid-water sampling (outside the bay), consisted of 103 pelagic, 3 demersal and 1 benthic (Table 1). A total of 21 different species were recognised. The number of specimens per species and total length ranges are shown in Table 2. Almost all the caught species, in particular, E. encrasicolus, Sardina pilchardus, Boops boops, Mullus barbatus and Illex coindetii, are typical of the Mediterranean Sea and are commercially relevant to Italian fishing (Irepa, 2010). Only one specimen was found to belong to a socalled alien species, specifically Sphyraena sphyraena. This is a typical species of the tropical seas, today present also in the Mediterranean Sea (Streftaris and Zenetos, 2006).

\subsection{Total mercury concentrations ( $\mathrm{HgT})$}

Total mercury concentrations measured in tissues from pelagic, demersal and benthic fishes, caught inside and outside of Augusta Bay, are graphically summarised in Fig. 2a and b. Mercury mean values calculated for each species, together with available comparative data from the literature and HgT content measured in anchovies from Marsala, are presented in Table 2.

Mercury concentrations ranged between 0.021 and $2.709 \mu \mathrm{g} \mathrm{g}^{-1}$ in muscles (Fig. 2a) and between 0.029 and $9.720 \mu \mathrm{g} \mathrm{g}^{-1}$ in livers (Fig. 2b). The HgT content in liver is from 1.5 to 6 times higher than that measured in muscles from the same specimens (Table 2). The highest HgT values were found in species caught inside the bay: 2 demersal specimens, a specimen of Diplodus vulgaris $\left(\mathrm{HgT}\right.$ in liver $\left.=4.979 \mu \mathrm{g} \mathrm{g}^{-1}\right)$ (extreme point in Fig. $2 \mathrm{~b}$ ) and a specimen of Serranus scriba ( $\mathrm{HgT}$ in muscle $=2.709 \mu \mathrm{g} \mathrm{g}^{-1}$ ) (extreme point in Fig. 2a), a large pelagic specimen of $S$. sphyraena ( $\mathrm{HgT}=9.720$ and $2.269 \mu \mathrm{g} \mathrm{g}^{-1}$ in liver and muscle, respectively) (Table 2) and a benthic specimen of Murena helena ( $\mathrm{HgT}=2.638 \mu \mathrm{g} \mathrm{g}^{-1}$ in muscle) (Table 2 ). However, these very high levels represent outliers of the whole dataset (Fig. $2 a$ and b). The highest non-outlier values refer once again to specimens caught inside the bay and specifically to benthic species (Fig. 2a and b). In particular, Scorpanea scrofa and Scorpanea notata show the highest HgT mean concentrations for both liver (1.638 and $2.339 \mu^{-1} \mathrm{~g}^{-1}$, respectively) and muscle (1.082 and $1.341 \mu \mathrm{g} \mathrm{g}^{-1}$, respectively) (Table 2). The lowest non-outlier ranges were found in pelagic specimens caught outside the bay $\left(0.021-0.167 \mu \mathrm{g} \mathrm{g}^{-1}\right.$ for muscles and 0.029-0.5708 for livers) (Fig. 2a and b), and the HgT mean values measured in the different studied species are substantially comparable (Table 2). Finally, data for demersal species from the 


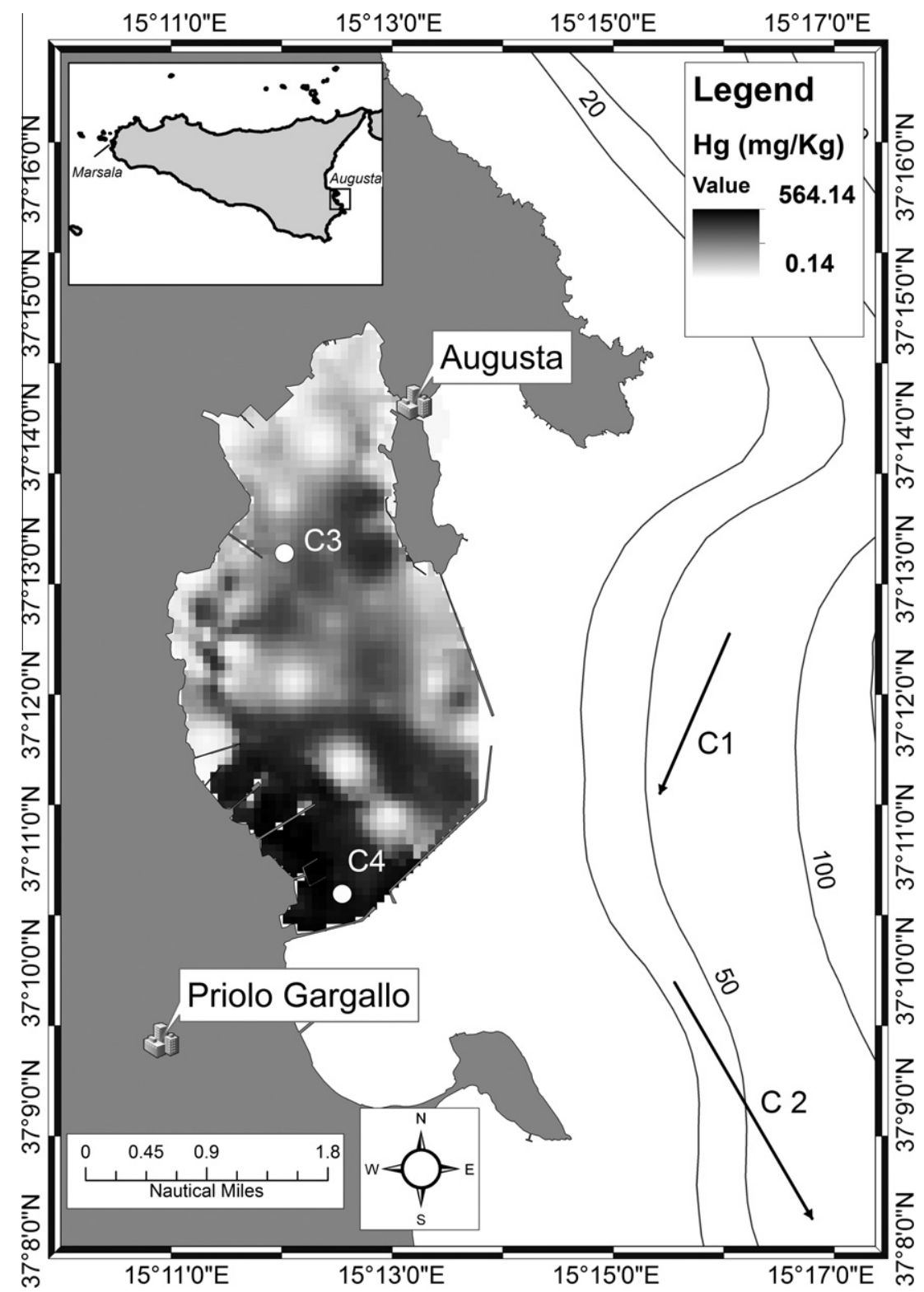

Fig. 1. Sampling sites in Augusta Bay and distribution of total mercury (HgT) in bottom sediments (data from Sprovieri et al. (2011)).

Table 1

Number of specimens per species caught in the sampling sites.

\begin{tabular}{|c|c|c|c|c|c|c|c|}
\hline \multicolumn{4}{|c|}{ Mid-water sampling (outside the bay) } & \multicolumn{4}{|c|}{ Bottom-water sampling (inside the bay) } \\
\hline Species & C1 (no.) & C2 (no.) & Habitat & Species & C3 (no.) & C4 (no.) & Habitat \\
\hline Engraulis encrasicolus & 20 & 20 & Pelagic & Diplodus annularis & 59 & 15 & Demersal \\
\hline Sardina pilchardus & 8 & 20 & Pelagic & Diplodus vulgaris & - & 3 & Demersal \\
\hline Boops boops & - & 20 & Pelagic & Pagellus erythrinus & 1 & 6 & Demersal \\
\hline Trachurus trachurus & - & 6 & Pelagic & Pagellus acarne & 11 & 1 & Demersal \\
\hline Illex coindetii & 6 & - & Pelagic & Sepia officinalis & 2 & 6 & Demersal \\
\hline Loligo forbesi & 3 & - & Pelagic & Serranus scriba & 2 & - & Demersal \\
\hline Pagellus erythrinus & 1 & - & Demersal & Caranx rhonchus & 1 & - & Pelagic \\
\hline Pagellus bogaraveo & 2 & & Demersal & Sphyraena sphyraena & 1 & & Pelagic \\
\hline \multirow[t]{6}{*}{ Mullus barbatus } & 1 & - & Benthic & Scorpaena notata & - & 5 & Benthic \\
\hline & & & & Scorpaena scrofa & 3 & 1 & Benthic \\
\hline & & & & Mullus barbatus & - & 3 & Benthic \\
\hline & & & & Mullus surmuletus & 1 & 1 & Benthic \\
\hline & & & & Murena helena & 1 & - & Benthic \\
\hline & & & & Octopus vulgaris & - & 1 & Benthic \\
\hline
\end{tabular}


Table 2

HgT means concentrations in muscle and liver of the analysed species and comparison with data for other areas.

\begin{tabular}{|c|c|c|c|c|c|c|c|c|}
\hline Species & No. & $\begin{array}{l}\text { Range of total length } \\
(\mathrm{mm})\end{array}$ & $\begin{array}{l}\text { HgT muscle } \\
\left(\mu \mathrm{g} \mathrm{g}^{-1}\right)\end{array}$ & S.D. & References & Site & $\begin{array}{l}\text { HgT liver } \\
\left(\mu \mathrm{g} \mathrm{g}^{-1}\right)\end{array}$ & S.D. \\
\hline \multirow{9}{*}{$\begin{array}{l}\text { Engraulis } \\
\quad \text { encrasicolus }\end{array}$} & 40 & $109-138$ & 0.052 & 0.019 & This work & Augusta & 0.204 & 0.147 \\
\hline & 11 & $120-139$ & 0.057 & 0.014 & This work & Marsala & 0.119 & 0.038 \\
\hline & & & 0.040 & & Bilandžić et al. (2011) & Adriatic sea & & \\
\hline & 9 & $121-147$ & 0.070 & 0.090 & Gibičar et al. (2009) & Adriatic sea $^{\mathrm{a}}$ & & \\
\hline & & & 0.030 & 0.030 & Copat et al. (2012) & Sicily (Catania) & & \\
\hline & & & 0.060 & 0.030 & Copat et al. (2012) & Syracuse (Sicily) & & \\
\hline & 18 & & 0.060 & & Pastor et al. (1994) & $\begin{array}{l}\text { Mediterranean sea } \\
(\text { Spain })^{\mathrm{a}}\end{array}$ & & \\
\hline & & & 0.070 & & Martorell et al. (2011) & $\begin{array}{l}\text { Mediterranean sea } \\
\text { (Spain) }\end{array}$ & & \\
\hline & 4 & & 0.055 & 0.003 & Tuzen (2009) & Black sea (Turkey) & & \\
\hline \multirow[t]{14}{*}{ Sardina pilchardus } & 28 & $115-150$ & 0.082 & 0.035 & This work & Augusta & 0.196 & 0.157 \\
\hline & 10 & $168-178$ & 0.090 & 0.040 & Gibičar et al. (2009) & Adriatic sea ${ }^{\mathrm{a}}$ & & \\
\hline & & & 0.080 & 0.030 & Copat et al. (2012) & Catania (Sicily) & & \\
\hline & & & 0.180 & & Buzina et al. (1995) & Adriatic sea ${ }^{\mathrm{a}}$ & & \\
\hline & & & 0.198 & & Buzina et al. (1995) & $\begin{array}{l}\text { Adriatic sea (Kastela } \\
\text { Bay) }\end{array}$ & & \\
\hline & 14 & & 0.052 & & Wolfgang (1983) & Adriatic sea ${ }^{a}$ & & \\
\hline & 35 & $190-260$ & 0.066 & & Wolfgang (1983) & Biscay Bay & & \\
\hline & 5 & $157-165$ & 0.050 & & Wolfgang (1983) & Mediterranean sea & & \\
\hline & 41 & & 0.170 & & Wolfgang (1983) & Ligurian sea & & \\
\hline & 28 & $120-150$ & 0.030 & & Wolfgang (1983) & North Africa (Ceuta) & & \\
\hline & 20 & $160-210$ & 0.040 & & Wolfgang (1983) & Western english channel & & \\
\hline & 38 & & 0.105 & & Pastor et al. (1994) & $\begin{array}{l}\text { Mediterranean sea } \\
(\text { Spain })^{\mathrm{a}}\end{array}$ & & \\
\hline & & & 0.019 & & Martorell et al. (2011) & $\begin{array}{l}\text { Mediterranean sea } \\
\text { (Spain) }\end{array}$ & & \\
\hline & 7 & $188-200$ & 0.033 & 0.016 & Harakeh et al. (1985) & Lebanon & & \\
\hline \multirow[t]{7}{*}{ Boops boops } & 20 & $95-150$ & 0.120 & 0.049 & This work & Augusta & 0.236 & 0.191 \\
\hline & 11 & $158-198$ & 0.196 & 0.204 & Hornung et al. (1980) & Israel $^{\mathrm{a}}$ & & \\
\hline & 1 & & 0.075 & & Pastor et al. (1994) & 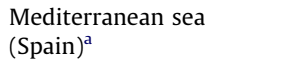 & & \\
\hline & 2 & $130-160$ & 0.190 & & $\begin{array}{l}\text { Stoeppler and Nürnberg } \\
\text { (1979) }\end{array}$ & Med. sea (Dubrovnik) & & \\
\hline & & & 0.267 & & Buzina et al. (1995) & Adriatic sea & & \\
\hline & & & 0.312 & & Buzina et al. (1995) & $\begin{array}{l}\text { Adriatic sea (Kastela } \\
\text { Bay) }\end{array}$ & & \\
\hline & 16 & $139-171$ & 0.036 & 0.025 & Harakeh et al. (1985) & Lebanon & & \\
\hline \multirow{8}{*}{$\begin{array}{l}\text { Trachurus } \\
\text { trachurus }\end{array}$} & 6 & $56-222$ & 0.131 & 0.147 & This work & Augusta & 0.344 & 0.176 \\
\hline & 2 & $260-285$ & 0.170 & & $\begin{array}{l}\text { Stoeppler and Nürnberg } \\
\text { (1979) }\end{array}$ & North sea (German Bight) & & \\
\hline & & 170 & 0.170 & & Mikac et al. (1984) & $\begin{array}{l}\text { Adriatic sea (Kastela } \\
\text { Bay) }\end{array}$ & & \\
\hline & 37 & $130-236$ & 0.122 & 0.101 & Hornung et al. (1980) & Israel $^{\mathrm{a}}$ & & \\
\hline & 16 & $159-203$ & 0.045 & 0.019 & Harakeh et al. (1985) & Lebanon & & \\
\hline & & & 0.053 & & Martorell et al. (2011) & $\begin{array}{l}\text { Mediterranean sea } \\
\text { (Spain) }\end{array}$ & & \\
\hline & 4 & & 0.078 & 0.005 & Tuzen (2009) & Black Sea (Turkey) ${ }^{a}$ & & \\
\hline & 5 & & 0.053 & 0.012 & Keskin et al. (2007) & Marmara sea (Turkey) & & \\
\hline Diplodus annularis & 74 & $109-179$ & 0.557 & 0.303 & This work & Augusta & 1.195 & 0.827 \\
\hline & & & 0.653 & & Buzina et al. (1995) & Adriatic sea ${ }^{a}$ & & \\
\hline & & & 0.628 & & Buzina et al. (1995) & $\begin{array}{l}\text { Adriatic sea (Kastela } \\
\text { Bay) }\end{array}$ & & \\
\hline Diplodus vulgaris & 3 & $102-179$ & 0.643 & 0.614 & This work & Augusta & 2.035 & 2.554 \\
\hline & 5 & & 0.378 & 0.017 & Keskin et al. (2007) & Marmara sea (Turkey) & & \\
\hline \multirow{2}{*}{$\begin{array}{l}\text { Sphyraena } \\
\text { sphyraena }\end{array}$} & 1 & 1190 & 2.269 & & This work & Augusta & 9.727 & \\
\hline & 14 & $219-295$ & 0.167 & 0.068 & Hornung et al. (1980) & Israel $^{\mathrm{a}}$ & & \\
\hline Caranx rhonchus & 1 & 264 & 1.701 & & This work & Augusta & & \\
\hline \multirow[t]{3}{*}{ Pagellus acarne } & 12 & $149-161$ & 0.254 & 0.028 & This work & Augusta & 0.618 & 0.178 \\
\hline & 3 & $135-141$ & 0.112 & & Hornung et al. (1980) & Israel $^{\mathrm{a}}$ & & \\
\hline & 15 & $164-182$ & 0.032 & 0.014 & Harakeh et al. (1985) & Lebanon & & \\
\hline Pagellus bogaraveo & 2 & $178-179$ & 0.266 & 0.227 & This work & Augusta & 1.230 & 0.700 \\
\hline \multirow[t]{4}{*}{ Pagellus erythrinus } & 8 & $154-205$ & 0.407 & 0.100 & This work & Augusta & 2.322 & 0.445 \\
\hline & 5 & 110 & 0.341 & 0.025 & Papetti and Rossi (2009) & Tyrrhenian sea (Lazio) & & \\
\hline & 57 & $115-187$ & 0.180 & 0.094 & Hornung et al. (1980) & Israel $^{\mathrm{a}}$ & & \\
\hline & 9 & $89-173$ & 0.240 & 0.190 & Gibičar et al. (2009) & Adriatic sea $^{\mathrm{a}}$ & & \\
\hline
\end{tabular}


Table 2 (continued)

\begin{tabular}{|c|c|c|c|c|c|c|c|c|}
\hline Species & No. & $\begin{array}{l}\text { Range of total length } \\
(\mathrm{mm})\end{array}$ & $\begin{array}{l}\text { HgT muscle } \\
\left(\mu \mathrm{g} \mathrm{g}^{-1}\right)\end{array}$ & S.D. & References & Site & $\begin{array}{l}\text { HgT liver } \\
\left(\mu \mathrm{g} \mathrm{g}^{-1}\right)\end{array}$ & S.D. \\
\hline & 28 & $140-152$ & 0.042 & 0.023 & Harakeh et al. (1985) & Lebanon & & \\
\hline & 5 & & 0.168 & & Pastor et al. (1994) & $\begin{array}{l}\text { Mediterranean sea } \\
(\text { Spain })^{\mathrm{a}}\end{array}$ & & \\
\hline & 5 & & 0.290 & 0.044 & Keskin et al. (2007) & Marmara sea (Turkey) ${ }^{a}$ & & \\
\hline \multirow[t]{2}{*}{ Serranus scriba } & 2 & $122-140$ & 2.165 & 0.768 & This work & Augusta & 2.581 & 0.592 \\
\hline & 3 & & 1.030 & 0.459 & Gibičar et al. (2009) & $\begin{array}{l}\text { Tyrrhenian sea } \\
\text { (Tuscany) }^{\mathrm{a}}\end{array}$ & & \\
\hline \multirow[t]{13}{*}{ Mullus barbatus } & 4 & $155-202$ & 0.815 & 0.777 & This work & Augusta & 1.518 & 0.582 \\
\hline & & $102-230$ & 0.116 & 0.056 & Hornung et al. (1980) & Israel $^{\mathrm{a}}$ & & \\
\hline & & & 0.400 & 0.400 & Storelli et al. (2004) & Ionian sea & & \\
\hline & & & 0.490 & 0.500 & Storelli et al. (2004) & Adriatic sea $^{a}$ & & \\
\hline & 13 & $117-180$ & 0.700 & 0.730 & Gibičar et al. (2009) & Adriatic sea $^{a}$ & & \\
\hline & & & 0.370 & & Buzina et al. (1995) & Adriatic sea & & \\
\hline & & & 0.318 & & Buzina et al. (1995) & Adriatic sea (Kastela & & \\
\hline & 59 & & 0.139 & & Pastor et al. (1994) & $\begin{array}{l}\text { Bay })^{\mathrm{a}} \\
\text { Mediterranean sea } \\
\text { (Spain) }\end{array}$ & & \\
\hline & & & 0.010 & & Martorell et al. (2011) & $\begin{array}{l}\text { Mediterranean sea } \\
\text { (Spain) }\end{array}$ & & \\
\hline & 30 & $128-166$ & 0.054 & 0.025 & Harakeh et al. (1985) & Lebanon & & \\
\hline & & $130-200$ & 0.233 & & $\begin{array}{l}\text { Stoeppler and Nürnberg } \\
\text { (1979) }\end{array}$ & $\begin{array}{l}\text { Mediterranean Sea } \\
\text { (Sardinia) }\end{array}$ & & \\
\hline & 4 & & 0.036 & 0.002 & Tuzen (2009) & Black Sea (Turkey) ${ }^{\mathrm{a}}$ & & \\
\hline & 5 & & 0.434 & 0.012 & Keskin et al. (2007) & Marmara sea (Turkey) ${ }^{a}$ & & \\
\hline \multirow[t]{5}{*}{ Mullus surmuletus } & 2 & $200-209$ & 0.662 & 0.089 & This work & Augusta & 1.112 & \\
\hline & 9 & $120-160$ & 0.086 & & Hornung et al. (1980) & Israel $^{\mathrm{a}}$ & & \\
\hline & 59 & & 0.139 & & Pastor et al. (1994) & $\begin{array}{l}\text { Mediterranean sea } \\
(\text { Spain })^{\mathrm{a}}\end{array}$ & & \\
\hline & 2 & 185-203 & 0.250 & & $\begin{array}{l}\text { Stoeppler and Nürnberg } \\
\text { (1979) }\end{array}$ & North sea (German Bight) & & \\
\hline & 37 & & 0.060 & & Bilandžic et al. (2011) & Adr.sea (Croatian coast) & & \\
\hline \multirow[t]{3}{*}{ Scorpaena scrofa } & 4 & $93-112$ & 1.082 & 0.285 & This work & Augusta & 1.637 & 0.380 \\
\hline & & & 0.222 & & Buzina et al. (1995) & Adriatic sea & & \\
\hline & & & 0.390 & & Buzina et al. (1995) & Adr. sea (Kastela Bay) ${ }^{a}$ & & \\
\hline \multirow[t]{2}{*}{ Scorpaena notata } & 5 & 114-133 & 1.340 & 0.380 & This work & Augusta & 2.339 & 0.529 \\
\hline & 5 & & 0.490 & 0.430 & Gibičar et al. (2009) & $\begin{array}{l}\text { Tyrrhenian sea } \\
\text { (Tuscany) }^{\mathrm{a}}\end{array}$ & & \\
\hline \multirow[t]{2}{*}{ Illex coindetii } & 6 & $33-92$ & 0.078 & 0.039 & This work & Augusta & & \\
\hline & 13 & $52-224$ & 0.100 & 0.100 & Gibičar et al. (2009) & Adriatic sea ${ }^{a}$ & & \\
\hline Loligo forbesi & 3 & $45-170$ & 0.147 & 0.024 & This work & Augusta & 0.311 & 0.011 \\
\hline Sepia officinalis & 8 & $108-148$ & 0.766 & 0.288 & This work & Augusta & & \\
\hline Octopus vulgaris & 1 & 123 & 0.443 & & This work & Augusta & & \\
\hline Murena helena & 1 & 800.5 & 2.638 & & This work & Augusta & 3.817 & \\
\hline
\end{tabular}

a Polluted site.

inner bay show the widest non-outlier ranges $\left(0.084-1.116 \mu \mathrm{g} \mathrm{g}^{-1}\right.$ for muscles, $0.109-2.747 \mu \mathrm{g} \mathrm{g}^{-1}$ for livers) and the most elevated number of outliers and extreme values (Fig. 2a and b). In particular, the highest $\mathrm{HgT}$ mean values $\left(2.165 \mu \mathrm{g} \mathrm{g}^{-1}\right.$ in liver and $2.581 \mu \mathrm{g} \mathrm{g}^{-1}$ in muscle) were measured in the S. scriba species (Table 2).

\subsection{THQ and EWI values}

Mean THQ and EWI values calculated for each caught species, inside and outside the bay, are reported in Table 3. Most of the fish species inside the bay show higher values (TQa $=1.53-15.8$; $\mathrm{TQb}=0.66-6.88 ; \mathrm{EWI}=1.06-11.0$ ) than those outside the studied area $(\mathrm{TQa}=0.31-4.20 ; \mathrm{TQb}=0.66-6.88 ; \mathrm{EWI}=0.22-2.91)$. In particular, the highest values were calculated for $M$. helena $(\mathrm{TQa}=15.8 ; \quad \mathrm{TQb}=6.88 ; \quad \mathrm{EWI}=11.0), \quad$ S. $\quad$ scriba $\quad(\mathrm{TQa}=13.0 ;$ $\mathrm{TQb}=5.65 ; \quad \mathrm{EWI}=9.02)$ and Caranx rhonchus $(\mathrm{TQa}=10.2$; $\mathrm{TQb}=4.44$; EWI = 7.09) caught inside the bay, while, the pelagic species outside the bay show the lowest values (TQa $=0.31-0.88$; $\mathrm{TQb}=0.14-0.38 ; \mathrm{EWI}=0.22-0.61$ ). Finally, no significant differ- ences were found between the same or similar species, collected inside (Pagellus acarne, Pagellus erythrinus, M. barbatus, Mullus surmuletus) and outside (Pagellus bogaraveo, P. erythrinus and M. barbatus) the bay.

\section{Discussion}

\subsection{Mercury bioaccumulation effects: length/age vs. HgT content}

The $\mathrm{Hg}$ accumulation in marine fish primarily depends on some important biokinetic parameters: assimilation from the ingested prey, uptake constants from the aqueous phase, de-toxification rate (Wang, 2012; Wang et al., 1997, 1998; Wang and Fisher, 1999; Dang and Wang, 2011) and environmental features (e.g., $\mathrm{Hg}$ concentration and speciation in seawater, dietary sources, etc.) (Wang and Wong, 2003). However, physiological and geochemical species-specific influences on $\mathrm{Hg}$ bioaccumulation are still not fully understood (Baines et al., 2002; Xu and Wang, 2002; Wang and Wong, 2003; Dang and Wang, 2012). 
a

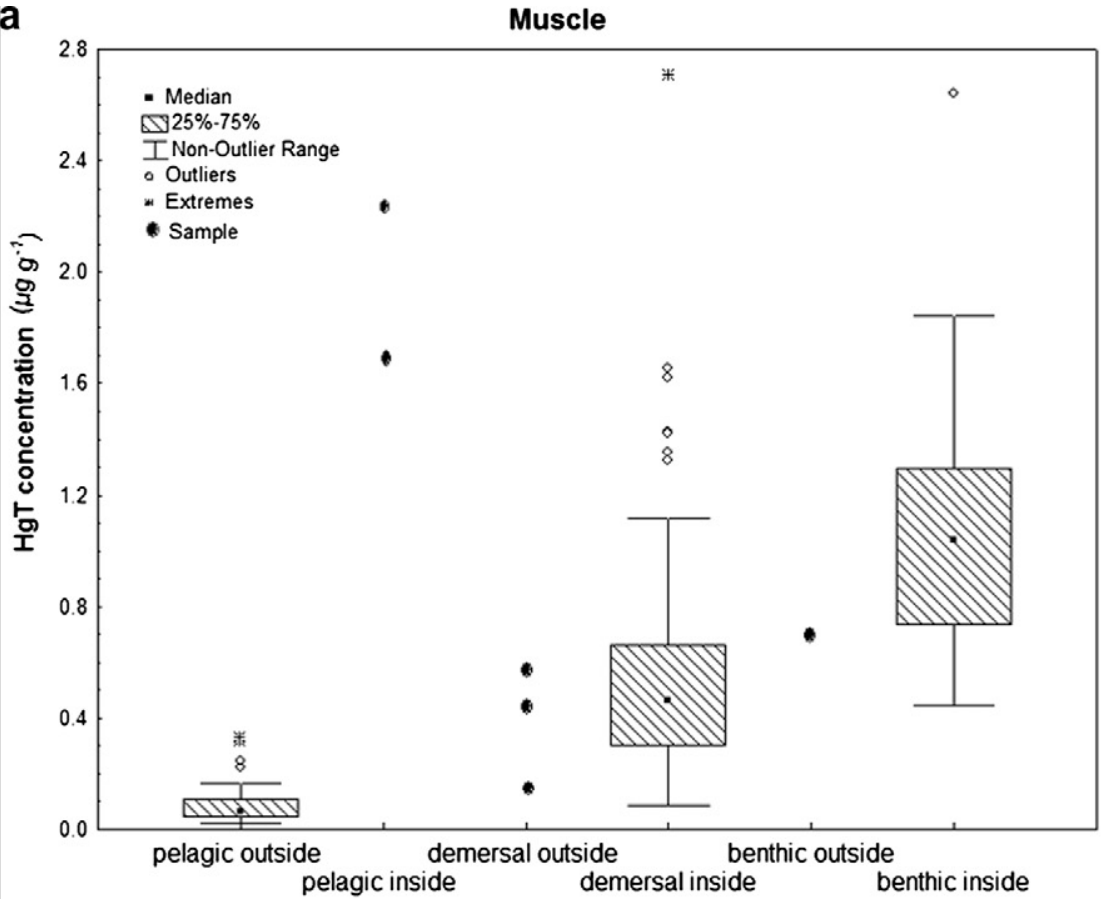

b

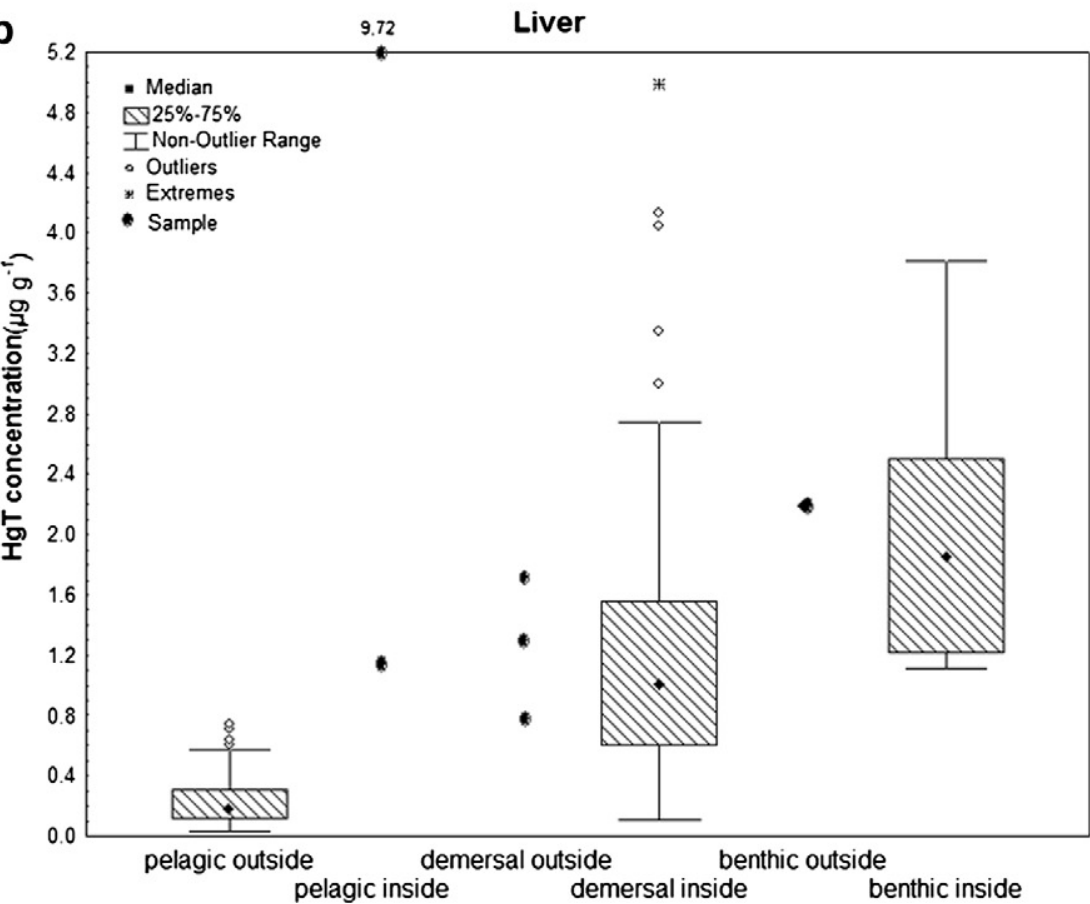

Fig. 2. Box-plots with HgT concentrations in the muscles and livers of pelagic, demersal and benthic fishes.

Several studies have demonstrated that $\mathrm{Hg}$ concentrations in the muscles of marine organisms proportionally increase with size and age (Lange et al., 1994; Burger et al., 2001; Green and Knutzen, 2003; Simonin et al., 2008). Moreover, Hg de-toxification rates appear negatively correlated with the fish size (Trudel and Rasmussen, 1997), supporting a potential correlation between $\mathrm{Hg}$ levels and size/age in the organisms. However, detailed investigations on different groups of species and on a wide range of HgT concentrations are lacking and, when available, sometimes controversial (Stafford and Haines, 2001), especially for fishes with low mercury levels (average below 0.2 ppm) (Park and Curtis, 1997; Burger and Gochfeld, 2011). Strong correlations between size and Hg levels in fish are reported for Swordfish (Xiphias gladius) and Bluefin Tuna (Thunnus thynnus) from the Mediterranean Sea (Storelli and Marcotrigiano, 2001), for several pelagic fish species from the Adriatic Sea (Storelli, 2008) and for S. pilchardus specimens from Tunisia (Joiris et al., 1999). Furthermore, Burger et al. (2007) found a positive correlation between size and $\mathrm{Hg}$ levels for 11 of 14 species of marine fishes collected in the western Aleutians (Bering Sea/North Pacific) and Luten et al. (1987) found the same positive correlation in Atlantic Cod. Moreover, Leonzio et al. (1981) report positive correlations between $\mathrm{Hg}$ content and weight in $\mathrm{M}$. barbatus and a slight $\mathrm{Hg}$ increasing trend with size in E. encrasicolus from the northern Tyrrhenian Sea. Finally, Gewurtz et al. (2011) show strong 
Table 3

THQ and EWI calculation for each caught species (inside and outside the Bay).

\begin{tabular}{|c|c|c|c|c|c|c|c|}
\hline \multicolumn{4}{|l|}{ Inside } & \multicolumn{4}{|l|}{ Outside } \\
\hline Species & THQa & THQb & EWI & Species & THQa & THQb & EWI \\
\hline Caranx rhonchus & 10.2 & 4.44 & 7.09 & Engraulis encrasicolus & 0.31 & 0.14 & 0.22 \\
\hline Diplodus annlularis & 3.34 & 1.45 & 2.32 & Sardina pilchardus & 0.49 & 0.21 & 0.34 \\
\hline Diplodus vulgaris & 3.86 & 1.68 & 2.68 & Boops boops & 0.72 & 0.31 & 0.50 \\
\hline Pagellus acarne & 1.53 & 0.66 & 1.06 & Trachurus trachurus & 0.79 & 0.34 & 0.55 \\
\hline Pagellus erythrinus & 2.30 & 1.00 & 1.60 & Illex coindetti & 0.47 & 0.20 & 0.33 \\
\hline Scophaena scrofa & 7.17 & 3.12 & 4.98 & Loligo forbesi & 0.88 & 0.38 & 0.61 \\
\hline Scorphaena notata & 8.05 & 3.50 & 5.59 & Pagellus bogaraveo & 1.60 & 0.69 & 1.11 \\
\hline Mullus barbatus & 5.59 & 2.43 & 3.88 & Pagellus erythrinus & 3.41 & 1.48 & 2.37 \\
\hline Mullus surmuletus & 3.97 & 1.73 & 2.76 & Mullus barbatus & 4.20 & 1.82 & 2.91 \\
\hline Serranus scriba & 13.0 & 5.65 & 9.02 & & & & \\
\hline Murena helena & 15.8 & 6.88 & 11.0 & & & & \\
\hline Octopus vulgaris & 2.66 & 1.16 & 1.85 & & & & \\
\hline Sepia officinalis & 4.60 & 2.00 & 3.19 & & & & \\
\hline
\end{tabular}

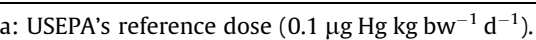

b: acceptable daily intake determined by WHO $\left(0.23 \mu \mathrm{g} \mathrm{Hg} \mathrm{kg} \mathrm{bw}^{-1} \mathrm{~d}^{-1}\right)$.

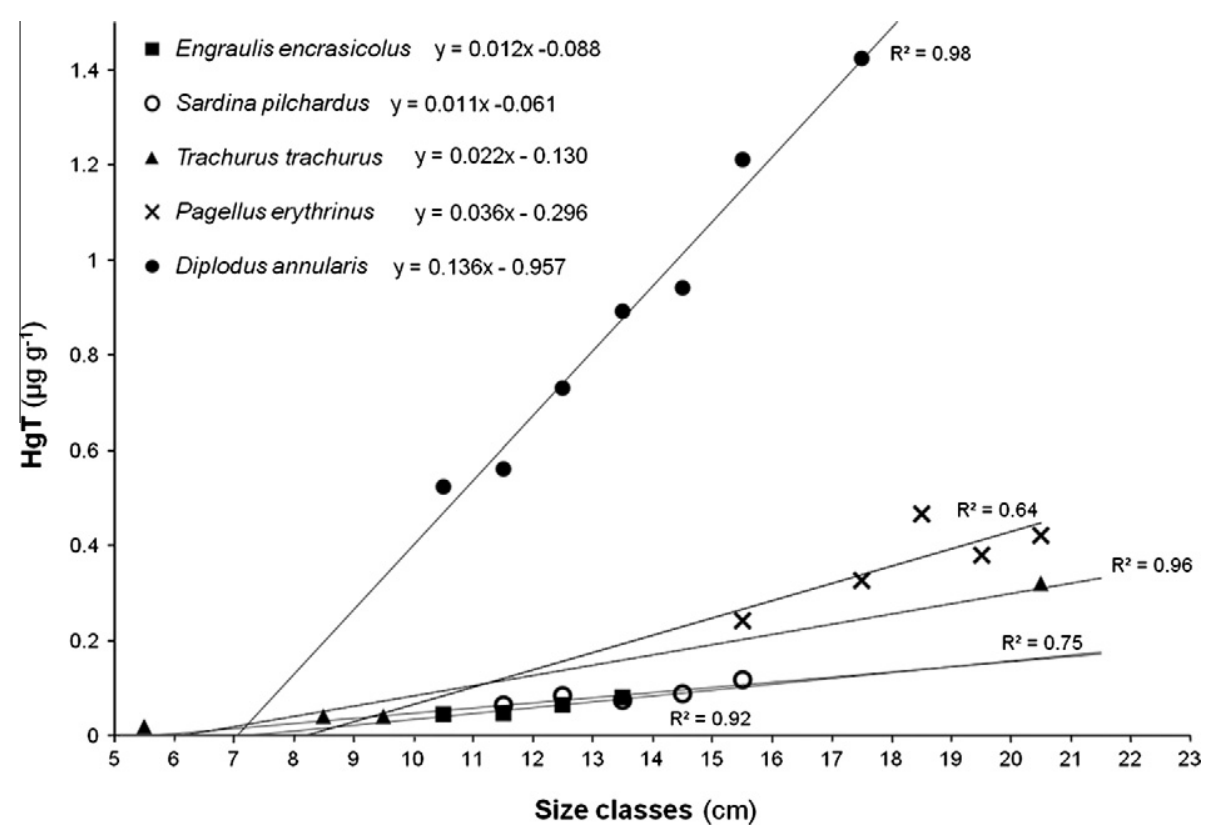

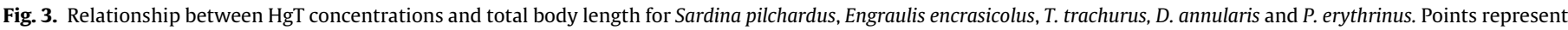
the mean values for each size class.

correlation between HgT concentration and length in most freshwater fishes from the Canadian Great Lakes and Ontario (Canada).

Here, the high number of specimens available from pelagic, benthic and demersal fish species associated with a wide range of length/age and HgT variability detected in tissues offer a challenging opportunity to explore in more depth the actual bioaccumulation processes of $\mathrm{Hg}$ in the studied organisms. In particular, we assumed the length of fishes as a reliable parameter for age estimates (Boening, 2000; Waldron and Kerstan, 2001; Scudder et al., 2009; Panfili et al., 2010; Basilone et al., 2011; Bacha et al., 2012) and, thus, reported HgT values vs. length to assess biomagnification of that contaminant with time. Statistically reliable and robust correlations were found between HgT mean values measured in muscles for size classes and length in $S$. pilchardus $\left(r^{2}=0.75\right), \quad$ E. encrasicolus $\left(r^{2}=0.92\right)$, Trachurus trachurus $\left(r^{2}=0.96\right)$, Diplodus annularis $\left(r^{2}=0.98\right)$ and $P$. erythrinus $\left(r^{2}=0.64\right)$ (Fig. 3). Specifically, the calculated HgT accumulation rates for S. pilchardus, E. encrasicolus, T. trachurus, P. erythrinus and $D$. annularis are $0.011,0.012,0.022,0.036$ and $0.136 \mu \mathrm{g} \mathrm{g}^{-1}$ $\mathrm{cm}^{-1}$, respectively, in good agreement with data reported by Hornung et al. (1980) for P. erythrinus and T. trachurus species. This definitively supports a significant linear HgT-length relationship for the studied fish species and a species-specific accumulation effect on the studied marine organisms.

In our dataset an evident increasing trend was measured between HgT content and age in the two most abundant species, $E$. encrasicolus and S. pilchardus (Fig. 4) with significant differences $(p<0.005$; ANOVA test) among age group, although, the restricted range of available age classes needs a larger data collection.

\subsection{Sources of $\mathrm{HgT}$ and fish contamination in Augusta Bay}

Muscles are the most commonly analysed tissues to monitor $\mathrm{Hg}$ levels in fishes because they represent the edible part of the organism associated with human health risk implications (Henry et al., 2004). Indeed, Hg accumulates over time more readily in liver than in muscle, but muscle appears to retain $\mathrm{Hg}$ for a much longer period (Boudou and Ribeyre, 1995). Thus, liver may provide information only on short-term exposure to $\mathrm{Hg}$ pollution or may bioaccumulate only when an organism is exposed to constant or increasing levels of dietary mercury (Atwell et al., 1998). This is clearly reflected in the studied dataset, where HgT concentration 


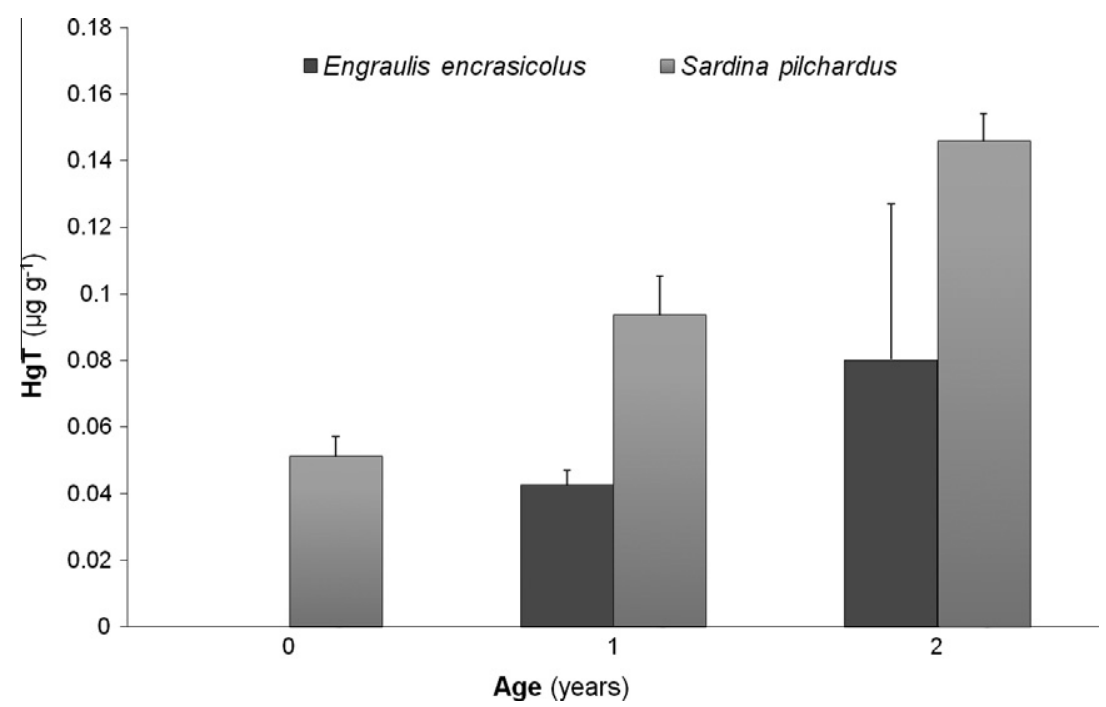

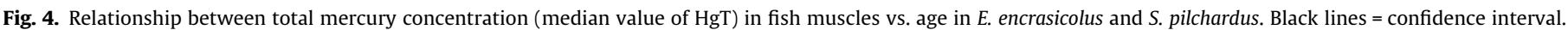

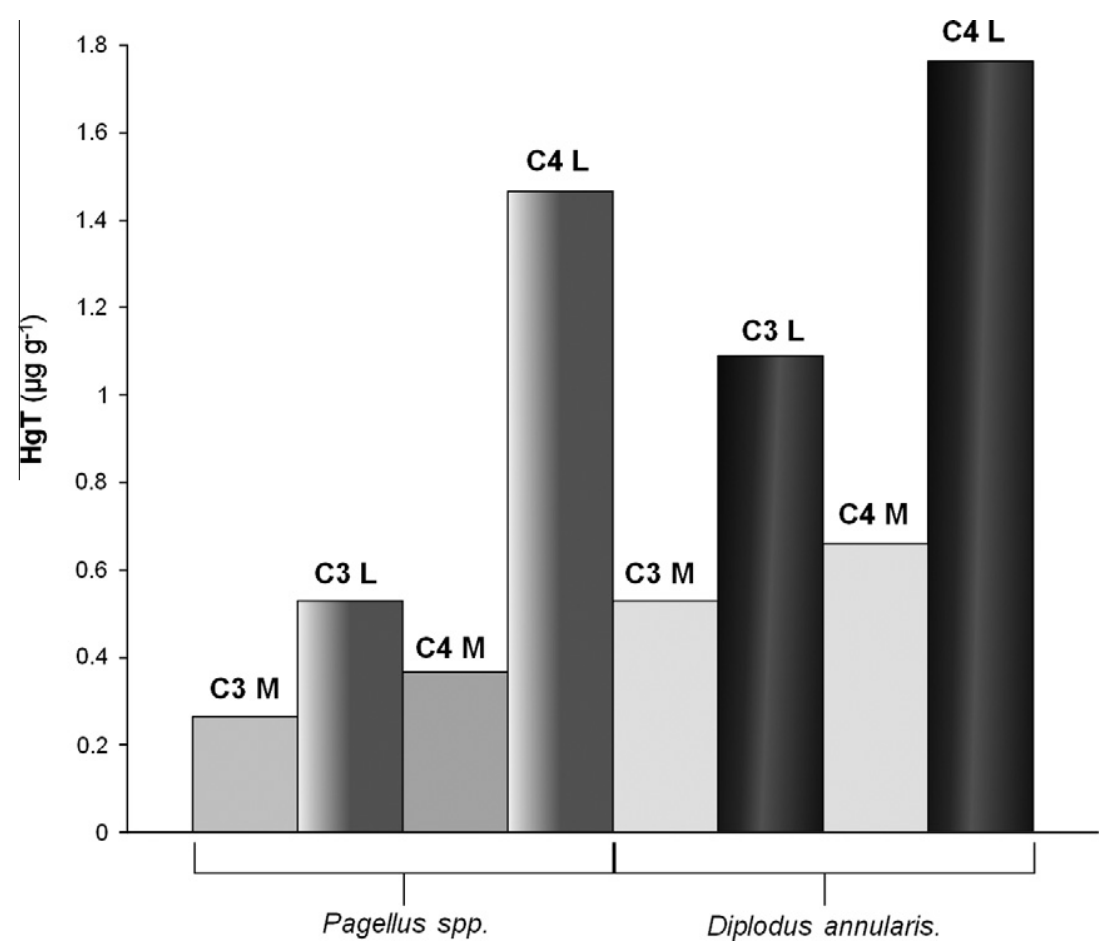

Fig. 5. Differences in muscle (M) and liver (L) HgT contents in Pagellus spp. and Diplodus annularis from the northern (C3) and the southern (C4) part of Augusta Bay.

measured in liver is up to two orders of magnitude higher than in muscles.

The HgT content measured in the tissues of fishes from Augusta Bay show an increasing trend with habitat depth, specifically, with highest values measured in benthic species with respect to the lowest levels detected in pelagic organisms (Table 2). Additionally, contamination effects show a south-north gradient evident from HgT levels measured on the ubiquitous Pagellus spp. and D. annularis specimens (Fig. 5). In particular, the highest HgT mean concentrations occur in fishes caught from southern Augusta Bay where bottom sediments show the highest concentrations of mercury (Sprovieri et al., 2011) (Fig. 1). This suggests a key role played by the highly polluted sediments as sources of $\mathrm{Hg}$ to the investigated marine environment. Also, measurements of $\mathrm{HgT}$ in seawater reported from the bottom, mid and surface waters by ENVIRON
International Team (2008) with an average concentration of $0.25 \mathrm{nmol} \mathrm{L}^{-1}$ and range of $0.05-0.37 \mathrm{nmol} \mathrm{L}^{-1}$ show a crucial effect of $\mathrm{Hg}$ efflux from sediments of the bay to the water column with a potential direct impact on the bioaccumulation processes in the trophic web. A direct comparison of HgT content in benthic species from Augusta Bay and other Mediterranean areas affected by comparable $\mathrm{Hg}$ discharges by chlor-alkali plant and sewage sludge disposal, specifically Tuscany and Israel (Hornung et al., 1980; Gibičar et al., 2009), show 2-7 times higher values, thus, underlying the combined effects of high pollution levels and specific biogeochemical pathways driving mercury bioavailability in the studied system (Table 2 ).

Sprovieri et al. (2011) and Fantozzi et al. (2013) have argued on a potential Hg export from Augusta Bay to the Eastern Mediterranean seawater, as a result of the measured 3D circulation system. 
Nonetheless, $\mathrm{Hg}$ contamination detected in sediments outside of Augusta Bay, by effects of dredged material from the inner bay (Di Leonardo et al., 2008; Tranchida et al., 2010), could also directly influence the state of pollution of the open sea.

Here, we extend the potential role of Augusta Bay as an $\mathrm{Hg}$ point source for the open ocean also considering the significant transfer of pollutants by pelagic fishes moving between the inner and external part of the bay. This implies potential effects on the food web of the surrounding area as already reported by several authors studying marine systems (Riisgard and Hansen, 1990; Futter, 1994; Jarman et al., 1996; Atwell et al., 1998). This hypothesis is supported by the high mean HgT concentrations measured in pelagic species caught outside the bay, which are similar to those reported for other sites affected by $\mathrm{Hg}$ pollution: the Adriatic Sea (Wolfgang, 1983; Storelli and Marcotrigiano, 2001; Storelli et al., 2002, 2004, 2007, 2010; Gibičar et al., 2009), Turkish areas (Tuzen, 2009), Spanish coastal areas (Pastor et al., 1994) and Israel area (Hornung et al., 1980) (Table 2).

The HgT mean concentrations measured in the livers of E. encrasicolus specimens from Augusta Bay are about twice as high $(p=0.044)$ as those measured in fishes from the unpolluted area of Marsala (Table 2), suggesting a direct, short-term effect of the bay pollution on the pelagic fishes. Moreover, the caught pelagic species prefer to inhabit warmer coastal seawaters during their first life stages (Basilone et al., 2011), but they generally move in deeper waters during the older stages (Wirszubski, 1953; Schneider, 1990; Whitehead, 1990), thus, representing a significant and potential vehicle of contaminants to the deep marine food web. This evidence definitively corroborates our hypothesis of a potential $\mathrm{Hg}$ export through the food web, from Augusta Bay to the surrounding area.

\subsection{Target hazard quotient and weekly intake: a real health risk from fishery in the Augusta Bay?}

Although estimation of the target hazard quotient (THQ) and weekly intake (EWI), do not provide a quantitative and definitive estimate on the dangerous health effects on exposed populations, these methodologies offer preliminary information on the health risk level resulting from pollutant exposure. Several authors showed that selenium (Se) offers protection against $\mathrm{Hg}$ toxicity (Parízek and Ostádalová, 1967; Satoh et al., 1985; Ralston, 2009; Lémire et al., 2010) that suggests to take in account Se contents in fishes to assess a real risk associated to $\mathrm{Hg}$ intake. Positive relationships has been found between $\mathrm{Hg}$ and Se contents in different seawater fishes (Burger and Gochfeld, 2011; Dang and Wang, 2011; Calatayud et al., 2012). Ralston et al. (2008) showed that $\mathrm{Se}: \mathrm{Hg}$ molar ratios above 1 protect against $\mathrm{Hg}$ toxicity. However this ratio definitively depends o species-specific toxic-kinetics processes (Watanabe, 2002; Burger and Gochfeld, 2012). This feature leads to a wide variability of Se:Hg molar ratios and makes difficult their use in risk assessment. Accordingly, here we estimated health risk for $\mathrm{Hg}$ intake only on THQ and EWI parameters. These indexes are widely used to assess risk associated with fish consumption (Storelli et al., 2004, 2010; Storelli, 2008; Martorell et al., 2011; Domingo et al., 2012). In particular, for no carcinogenic effects, an HQ exceeding 1.0 indicates a potential health risk (US EPA, 1989). In our dataset, either using USEPA's reference dose (TQa) that WHO acceptable daily intake (TQb), species inside the Bay exceeded the value 1 in all cases, while fishes outside the Bay only in demersal and benthic fishes ( $P$. bogaraveo, $P$. erythrinus, $M$. barbatus) (Table 3). International agencies indicate a provisional tolerable weekly intake (PTWI) of $\mathrm{Hg}$, ranging from $0.7 \mu \mathrm{g} \mathrm{kg}^{-1}$ body weight (b.w.) (US-EPA, 2004) to $1.6 \mu \mathrm{g} \mathrm{kg}^{-1}$ b.w. (FAO/WHO, 2006). These limits represent safe values for human population over lifetime. The calculated EWI index exceed the PTWI (US-
EPA, 2004; FAO/WHO) in almost all the species collected inside the Bay and in demersal and benthic fishes from outside. In summary, the calculated THQ and EWI highlight that the consumption of fish from inside the Bay represents a serious risk for human health of resident populations and confirm the importance of the current fishing ban in this area. Also, the results suggest caution in the consumption of fishes from outside the Bay, especially of demersal and benthic species, confirming that $\mathrm{Hg}$ contamination in this area is a serious concern that calls for appropriate and timely social actions.

\section{Conclusions}

The main conclusions of this work can be synthesised as follows:

- The high HgT concentrations measured in benthic species from Augusta Bay suggest an active release mechanism of mercury from polluted sediments to the water column, with consequent effects of bioaccumulation in the trophic web.

- High contamination of pelagic species measured in the external zone of the bay confirms the role of the Augusta marine environment as a potential $\mathrm{Hg}$ source for the surrounding area and underscores the crucial risk associated with contaminant transfer from the semi-enclosed basin to the open sea.

- The THQ and EWI values advise that consumption of fish from inside the Augusta Bay represents a serious risk for human health of the local populations, while suggest caution in consuming demersal and benthic fishes from outside the Augusta Bay definitively demanding for appropriate social actions.

\section{Conflict of Interest}

The authors declare that there are no conflicts of interest.

\section{Acknowledgements}

This research is part of an Italian project funded by the "Assessorato della Salute della Regione Siciliana". The authors would like to express sincere thanks to Dr. M.M. Uccello and Dr. G. Baffo (Zooprophylatic Institute of Augusta) for their facilities and support in fish sampling. Thanks are also due to Dr. F. Bulfamante (IAMC-CNR, Capo Granitola) for logistic contribution and Dr. M. Barra (IAMCCNR, Naples) for comments and suggestions on statistical methodologies. Three anonymous reviewers are warmly tanks for their contributions and suggestions.

\section{References}

Atwell, L., Hobson, K.A., Welch, H.E., 1998. Biomagnification and bioaccumulation of mercury in an arctic marine food web: insights from stable nitrogen isotope analysis. Can. J. Fish. Aquat. Sci. 55, 1114-1121.

Ausili, A., Gabellini, M., Cammarata, G., Fattorini, D., Benedetti, M., Pisanelli, B., Gorbi, S., Regoli, F., 2008. Ecotoxicological and human health risk in a petrochemical district of southern Italy. Mar. Environ. Res. 66, 215-217.

Bacha, M., Moali, A., Benmansour, N.E., Brylinski, J.M., Mahé, K., Amara, R., 2012. Relationships between age, growth, diet and environmental parameters for anchovy (Engraulis encrasicolus L.) in the Bay of Bénisaf (SW Mediterranean, west Algerian coast). Cybium 34 (1), 47-57.

Baines, S.B., Fisher, N.S., Stewart, R., 2002. Assimilation and retention of selenium and other trace elements from crustacean food by juvenile striped bass (Morone saxatilis). Limnol. Oceanogr. 47, 646-655

Basilone, G., Bonanno, A., Patti, B., Gancitano, S., Caruana, L., Bonsignore, M., Aronica, S., Tranchida, G., 2011. Juvenile Anchovy, Engraulis encrasicolus, Habitat Conditions and Daily Growth in the Central Mediterranean Sea. Marine research Fishery and Sea Resources, 2057. Volume Speciale Mare - CNR Dipartimento Terra e Ambiente.

Bianchi, F., Bianca, S., Linzalone, N., Madeddu, A., 2004. Epidemiol. Prev. 28, 87-93.

Bianchi, F., Bianca, S., Dardanoni, G., Linzalone, N., Pierini, A., 2006. Malformazioni congenite nei nati residenti nel Comune di Gela (Sicilia, Italia). Epidemiol. Prev. 30 (1), 19-26. 
Bilandžić, N., Đokić, M., Sedak, M., 2011. Metal content determination in four fish species from the Adriatic Sea. Food Chem. 124, 1005-1010.

Boening, D.W., 2000. Ecological effects, transport, and fate of mercury: a general review. Chemosphere 40, 1335-1351.

Boudou, A., Ribeyre, F., 1995. Mercury in the food web: accumulation and transfer mechanisms. In: Sigel, A., Sigel, H. (Eds.), Metal Ions in Biological Systems. Marcel Dekker, Inc., New York, pp. 289-319.

Burger, J., Gochfeld, M., 2011. Mercury and selenium levels in 19 species of saltwater fish from New Jersey as a function of species, size, and season. Sci. Total Environ. 409, 1418-1429.

Burger, J., Gochfeld, M., 2012. Selenium and mercury molar ratios in saltwater fish from New Jersey: individual and species variability complicate use in human health fish consumption advisories. Environ. Res. 114, 12-23.

Burger, J., Gaines, K.F., Gochfeld, M., 2001. Ethnic differences in risk from mercury among Savannah River fishermen. Risk Anal. 21, 533-544.

Burger, J., Gochfeld, M., Jeitner, C., Burke, S., Stamm, T., Snigaroff, R., 2007. Mercury levels and potential risk from subsistene foods from the Aleutians. Sci. Total Environ. 384, 93-105.

Buzina, R., Stegnar, P., Buzina- Suboticanec, K., Horvat, M., Petric, I., Farley, T.M.M. 1995. Dietary mercury intake and human exposure in an Adriatic population. Sci. Total Environ. 170, 199-208.

Calatayud, M., Devesa, V., Virseda, J.R., Barberá, R., Montoro, R., Vélez, D., 2012. Mercury and selenium in fish and shellfish: occurrence, bioaccessibility and uptake by Caco-2 cells. Food Chem. Toxicol. 50, 2696-2702.

Campana, S.E., Gagnè, J.A., Munro, J., 1987. Otolith microstructure of Larval Herring (Clupea harengus): image or reality? Can. J. Fish. Aquat. Sci. 44, 1922-1929.

Carbonell, G., Bravo, J.C., Fernàndez, C., Tarazona, J.V., 2009. A new method for total mercury and methyl mercury analysis in muscle of seawater fish. Bull. Environ. Contam. Toxicol. 83, 210-213.

Copat, C., Bella, F., Castaing, M., Fallico, R., Sciacca, S., Ferrante, M., 2012. Heavy metals concentrations in fish from Sicily (Mediterranean Sea) and evaluation of possible health risks to consumers. Bull. Environ. Contam. Toxicol. 88, 78-83.

Dang, F., Wang, W.X., 2011. Antagonistic interaction of mercury and selenium in a marine fish is dependent on their chemical species. Environ. Sci. Technol. 45, 3116-3122.

Dang, F., Wang, W.X., 2012. Why mercury concentration increases with fish size? Biokinetic explanation. Environ. Pollut. 163, 192-198.

Das, S.K., Sharma, A., Talukder, G., 1982. Effects of mercury on cellular systems in mammals - a review. Nucleus (Calcutta) 25, 193-230.

De Flora, S., Bennicelli, C., Bagnasco, M., 1994. Genotoxicity of mercury compounds: a review. Mutat. Res. 317, 57-79.

Di Leonardo, R., Bellanca, A., Capotondi, L., Cundy, A., Neri, R., 2007. Possible impacts of $\mathrm{Hg}$ and $\mathrm{PAH}$ contamination on benthic foraminiferal assemblages: an example from the Sicilian coast, central Mediterranean. Sci. Total Environ. $388,168-183$.

Di Leonardo, R., Bellanca, A., Angelone, M., Leonardi, M., Neri, R., 2008. Impact of human activities on the central Mediterranean offshore: evidence from $\mathrm{Hg}$ distribution in box-core sediments from the Ionian Sea. Appl. Geochem. 23, 3756-3766.

Domingo, J.L., Perelló, G., Bordonaba, J.G., 2012. Dietary intake of metals by the population of tarragona County (Catalonia, Spain): results from a duplicate. Biol. Trace Elem. Res. 146, 420-425.

Elhassani, S.B., 1983. The many faces of methylmercury poisoning. J. Toxicol. 19, 875-906.

ENVIRON International Team, 2008. Appendix A Sediment Investigation Activities Performed by ENVIRON Summer and Fall 2008 Augusta Bay, Sicily, Italy.

Fano, V., Cernigliano, A., Scondotto, S., 2005. Stato di salute nella popolazione delle aree a rischio di crisi ambientale e nei siti di interesse nazionale della Sicilia: analisi della mortalità (aa 1995-2000) e dei ricoveri ospedalieri (aa 20012003). O.E. Notiziario della Regione Sicilia Assessorato Sanità (numero monografico).

Fano, V., Cernigliano, A., Scondotto, S., Pollina Addario, S., Caruso, S., Mira, A., Forastiere, F., Perucci, C.A., 2006. Analisi della mortalità (1995-2000) e dei ricoveri ospedalieri (2001-2003) nell'area industriale di Gela. Epidemiol. Prev. 30 (1), 27-32.

Fantozzi, L., Manca, G., Ammoscato, I., Pirrone, N., Sprovieri, F., 2013. The cycling and sea-air exchange of mercury in the waters of the Eastern Mediterranean during the 2010 MED-OCEANOR cruise campaign. Sci. Total Environ. 448, 151-162.

FAO (Food and Agriculture Organization of the United Nations/World Health Organization), 2005. Statistical databases. <http://faostat.fao.org> (accessed 10.12.12).

Ficco, P., Cammarata, G., Bacci, E., 2009. Procedimento n. 5010/08 RGNR. Relazione di consulenza tecnica in merito all'origine dei contaminanti presenti nei sedimenti ed alle strategie per il risanamento della Rada di Augusta. Per conto della Procura della Repubblica presso il Tribunale ordinario di Siracusa., pp. 131

Futter, M.N., 1994. Pelagic food-web structure influences probability of mercury contamination in lake trout (Salvelinus namaycush). Sci. Total Environ. 145, 712 .

Gewurtz, S.B., Bhavsar, S.P., Fletcher, R., 2011. Influence of fish and sex on mercury/ PCB concentration importance for fish consumption advisories. Environ. Int. 37 425-434.

Gibičar, D., Horvat, M., Logar, M., Fajon, V., Falnoga, I., Ferrara, R., Lanzillotta, E., Ceccarini, C., Mazzolai, B., Denby, B., Pacyna, J., 2009. Human exposure to mercury in the vicinity of chlor-alkali plant. Environ. Res. 109, 355-367.
Gilmour, C.C., Henry, E.A., 1991. Mercury methylation in aquatic systems affected by acid deposition. Environ. Pollut. 71 (2-4), 131-169.

Green, N.W., Knutzen, J., 2003. Organohalogens and metals in marine fish and mussels and some relationships to biological variables at reference localities in Norway. Mar. Pollut. Bull. 46, 362-377.

Harakeh, M.S., Acra, A., Jurdi, M., Karahagopian, Y., 1985. Mercury levels in some species of fish from the coast of lebanon. Mar. Environ. Res. 16, 13-22.

Henry, F., Amara, R., Courcot, L., Lacouture, D., Bertho, M.L., 2004. Heavy metals in four fish species from the Southern Bight of the North Sea. Environ. Int. 30, 675683

Holsbeek, L., Das, H.K., Joiris, C.R., 1996. Mercury in human hair and relation to fish consumption in Bangladesh. Sci. Total Environ. 186, 181-188.

Hornung, H., Zismann, L., Oren, O.H., 1980. Mercury in 12 Mediterranean Trawl fishes of Israel. Environ. Int. 3, 243-248.

Horvat, M., Covelli, S., Faganeli, J., Logar, M., Mandić, V., Rajar, R., Širca, A., Žagard, D. 1999. Mercury in contaminated coastal environments; a case study: the Gulf of Trieste. Sci. Total Environ. 237 (238), 43-56.

ICRAM, 2005. Valutazione preliminare dei dati della caratterizzazione ambientale della rada di augusta - aree prioritarie ai fini della messa in sicurezza di emergenza BOI-PR-SI-GP-RADA DI AUGUSTA-01.02.

ICRAM, 2008. Istituto Centrale Per La Ricerca Scientifica E Tecnologica Applicata Al Mare, Progetto preliminare di bonifica dei fondali della rada di Augusta nel sito di interesse nazionale di Priolo - Elaborazione definitiva, BoI-Pr-SI-PR-Rada di Augusta-03.22, pp. 182.

IREPA, 2010. Osservatorio economico sulle strutture produttive della pesca marittima in Italia 2010. Edizioni Scientifiche Italiane, Napoli, 2011, pp. 120 (accessed 3.12.12)

Jarman, W.M., Hobson, K.A., Sydeman, W.J., Bacon, C.E., McLaren, E.B., 1996. Influence of trophic position and feeding location on contaminant levels in the Gulf of the Farallones food web revealed by stable isotope analysis. Environ. Sci. Technol. 30, 654-660.

Joiris, C.R., Holsbeek, L., Moatemri, N.L., 1999. Total and methylmercury in Sardines Sardinella aurita and Sardina pilchardus from Tunisia. Mar. Pollut. Bull. 38 (3), 188-192.

Keskin, Y., Baskaya, R., Özyaral, O., Yurdun, T., Lüleci, N.E., Hayran, O., 2007. Cadmium, lead, mercury and copper in fish from the Marmara sea, Turkey. Bull. Environ. Contam. Toxicol. 78, 258-261.

La Mesa, M., Donato, F., Giannetti, G., Arneri, E., 2009. Growth and mortality rates of European anchovy (Engraulis encrasicolus) in the Adriatic Sea during the transition from larval to juvenile stages. Fish. Res. 96, 275-280.

Lange, T.R., Royals, H.E., Connor, L.L., 1994. Mercury accumulation in largemouth bass (Micropterus salmoides) in a Florida lake. Arch. Environ. Contam. Toxicol. 27, 466-471.

Le Donne, K., Ciafani, S., 2008. Monitoraggio dell'inquinamento atmosferico da mercurio nei principali impianti cloro-soda italiani. Ing. Ambientale. 37, 4552.

Lémire, M., Fillion, M., Frenette, B., Mayer, A., Philibert, A., Passos, C.J.S., Guimarães, J.R.D., Barbosa Jr., F., Mergler, D., 2010. Selenium and mercury in the Brazilian Amazon: opposing influences on age-related cataracts. Environ. Health Perspect. 118 (11), 1584-1589.

Leonzio, C., Bacci, E., Focardi, S., Renzoni, A., 1981. Heavy metals in organisms from the northern Tyrrhenian sea. Sci. Total Environ. 20, 131-146.

Luten, J.B., Bouquet, W., Riekwel-Booy, G., Rauchbaar, A.B., Scholte, M.W.M., 1987. Mercury in flounder, Platichtys flesus, cod, Gadus morhua, and perch, Perca fluviatilis, in relation to their length and environment. Bull. Environ. Contam. Toxicol. 38, 318-323.

Madeddu, A., Contrino, L., Tisano, F., Sciacca, S., Milisenna, V., 2006. Rerum Cognoscere Causas (Atlante della Mortalità e dei Ricoveri per i tumori e per la patologie cronico degenerative in Provincia di Siracusa 2003-2005).

Martorell, I., Perelló, G., Martí-Cid, R., Llobet, J.M., Castell, V., Domingo, J.L., 2011. Human exposure to arsenic, cadmium, mercury, and lead from foods in Catalonia, Spain: temporal trend. Biol. Trace Elem. Res. 142, 309-322.

Martuzzi, M., Mitis, F., Biggeri, A., Terracini, B., Bertollini, R., 2006. Ambiente e stato di salute nella popolazione delle aree a rischio di crisi ambientale in Italia. Epidemiol. Prev. 26 (6 Suppl), 1-53.

Mason, R.P., Fitzgerald, W.F., 1990. Alkylmercury species in the equa-torial Pacific. Nat. London 347, 457-459.

Mason, R.P., Fitzgerald, W.F., 1991. Mercury speciation in open ocean waters. Water Air Soil Pollut. 56, 745-767.

Mikac, N., Picer, M., Stegnar, P., Žnidarić, T., 1984. Mercury distribution in a polluted marine area, ratio of total mercury, methyl mercury and selenium in sediments, mussels and fish. Water Res. 19 (I1), 1387-1392.

Nakagawa, R., Yumita, Y., Hiromoto, M., 1997. Total mercury intake from fish and shellfish by Japanese people. Chemosphere 35, 2909-2913.

Nielsen, J.D., 1992. Sources of error in otolith microstructure examination. Can. Special Pub. Fish. Aquat. 117, 115-125.

Panfili, M., Donato, F., Morello, E.B., Arneri, E., 2010. Growth rates of early life stages of Engraulis encrasicolus and Sardina pilchardus in the Adriatic sea (Italy). Rapp. Commun. Int. Mer. Médit., 39.

Papetti, P., Rossi, G., 2009. Heavy metals in the fishery products of low Lazio and the use of metallothionein as a biomarker of contamination. Environ. Monit. Assess. 159, 589-598.

Parízek, J., Ostádalová, I., 1967. The protective effect of small amounts of selenite in sublimate intoxication. Experientia 23, 142-143. 
Park, J.G., Curtis, L.R., 1997. Mercury distribution in sediments and bioaccumulation by fish in two Oregon reservoirs: point-source and nonpoint source impacted systems. Arch. Environ. Contam. Toxicol. 33, 423-429.

Pastor, A., Hernandez, F., Peris, M.A., Beltran, J., Sancho, J.V., Castillo, M.T., 1994. Levels of heavy metals in some marine organisms from the Western Mediterranean Area (Spain). Mar. Poll. Bull. 28 (1), 50-53.

Ralston, N.V.C., 2009. Introduction to 2nd issue on special topic: selenium and mercury as interactive environmental indicators. Environ. Bioindic. 4, 286-290.

Ralston, N.V.C., Ralston, C.R., Blackwell III, J.L., Raymond, L.J., 2008. Dietary and tissue selenium in relation to methylmercury toxicity. Neurotoxicology 29, 802-811.

Renzoni, A., Zino, F., Franchi, E., 1998. Mercury levels along the food chain and risk for exposed populations. Environ. Res. 77, 68-72.

Riisgard, H.U., Hansen, S., 1990. Biomagnification of mercury in a marine grazing food-chain: algal cells Phaeodactylum tricorntum, mussel Mytilus edulis and flounders Platichthys flesus studied by means of a stepwise-reduction-CVAA method. Mar. Ecol. Prog. Ser. 62, 259-270.

Satoh, H., Yasuda, N., Shimai, S., 1985. Development of reflexes in neonatal mice prenatally exposed to methylmercury and selenite. Toxicol. Lett. 25, 199-203.

Schneider, W., 1990. FAO species identification sheets for fishery purposes. Field Guide to the Commercial Marine Resources of the Gulf of Guinea. Prepared and Published with the Support of the FAO Regional Office for Africa, FAO, Rome. pp. 268.

Scudder, B.C., Chaser, L.C., Wentz, D.A., Bauch, N.J., Brigham, M.E., Moran, P.W., 2009. Mercury in fish, bed sediment, and water from streams across the United States, 1998-2005. US Dept of Interior, Report 2009-5109, Reston, Virginia, pp. 74

Simonin, H.A., Loukmas, J.J., Skinner, L.C., Roy, K.M., 2008. Lake variability: key factors controlling mercury concentrations in New York state fish. Environ. Pollut. 154, 107-115.

Sprovieri, M., Oliveri, E., Di Leonardo, R., Romano, E., Ausili, A., Gabellini, M., Barra, M., Tranchida, G., Bellanca, A., Neri, R., Budillon, F., Saggiomo, R., Mazzola, S., Saggiomo, V., 2011. The key role played by the Augusta basin (southern Italy) in the mercury contamination of the Mediterranean sea. J. Environ. Monit. 13, 1753.

Stafford, C.P., Haines, T.A., 2001. Mercury contamination and growth rate in two piscivore populations (2001). Environ. Toxicol. Chem. 20, 2099-2101.

Stoeppler, M., Nürnberg, H.W., 1979. Comparative studies on trace metal levels in marine biota III. Typical levels and accumulation of toxic trace metals in muscle tissue and organs of marine organisms from different European seas. Ecotoxicol. Environ. Safe. 3, 335-351.

Storelli, M.M., 2008. Potential human health risks from metals ( $\mathrm{Hg}, \mathrm{Cd}$, and $\mathrm{Pb}$ ) and polychlorinated biphenyls (PCBs) via seafood consumption: estimation of target hazard quotients (THQs) and toxic equivalents (TEQs). Food Chem. Toxicol. 46, 2782-2788.

Storelli, M.M., Marcotrigiano, G.O., 2001. Total mercury levels in muscle tissue of swordfish (Xiphias glaudius) and bluefin tuna Thunnus thynnus) from the Mediterranean sea. J. Food Protect. 64, 1058-1061.

Storelli, M.M., Stuffler, R.G., Marcotrigiano, G.O., 2002. Total and methylmercury residues in tunafish from the Mediterranean sea. Food Addit. Contam. 19, 715720.

Storelli, M.M., Storelli, A., Giacominelli-Stuffler, R., Marcotrigiano, G.O., 2004 Mercury speciation in the muscle of two commercially important fish, hake (Merluccius merluccius) and striped mullet (Mullus barbatus) from the Mediterranean sea: estimated weekly intake. Food Chemistry 89 (2005), 295300.

Storelli, M., Barone, G., Piscitelli, G., Marcotrigiano, G.O., 2007. Mercury in fish: concentrations vs. fish size and estimates of mercury intake. Food Addit Contam. 24, 1353-1357.
Storelli, M.M., Barone, G., Cuttone, G., Giungato, D., Garofalo, R., 2010. Occurrence of toxic metals $(\mathrm{Hg}, \mathrm{Cd}$ and $\mathrm{Pb})$ in fresh and canned tuna: public health implications. Food Chem. Toxicol. 48, 3167-3170.

Streftaris, N., Zenetos, A., 2006. Alien marine species in the Mediterranean - the 100 'Worst Invasives' and their impact. Med. Mar. Sci. 7 (1), 87-118.

Tomasello, B., Copat, C., Pulvirenti, V., Ferrito, V., Ferrante, M., Renis, M., Sciacca, S. Tigano, C., 2012. Biochemical and bioaccumulation approaches for investigating marine pollution using Mediterranean rainbow wrasse, Coris julis (Linneaus 1798). Ecotoxicol. Environ. Safe. 86, 168-175.

Tranchida, G., Bellanca, A., Angelone, M., Bonanno, A., Langone, L., Mazzola, S., Neri, R., Patti, B., 2010. Chronological records of metal deposition in sediments from the Strait of Sicily, central Mediterranean: assessing natural fluxes and anthropogenic alteration. J. Mar. Syst. 79, 157-172.

Trudel, M., Rasmussen, J.B., 1997. Modeling the elimination of mercury by fish. Environ. Sci. Technol. 31, 1716-1722.

Tuzen, M., 2009. Toxic and essential trace elemental contents in fish species from the Black sea, Turkey. Food Chem. Toxicol. 47, 1785-1790.

Uriarte, A., Dueñas, C., Duhamel, E., Grellier, P., Rico, I., Villamor, B., 2007, 2006 Anchovy Otolith Workshop. Working Document to the 2007 ICES Planning Group on Commercial Catch, Discards and Biological Sampling (PGCCDBS).

US EPA, 1989. Risk Assessment Guidance for Superfund: Human Health Evaluation Manual (Part A), Interim Final, December.

US EPA, 2004. Origin of the 1 meal/week noncommercial fish consumption rate in national advisory for mercury. Office of Water, National Fish and Wildlife Contamination Program <http://cfpub.epa.gov> (accessed 10.12.12).

Waldron, M.E., Kerstan, M., 2001. Age validation in horse mackerel (Trachurus trachurus) otoliths. ICES J. Mar. Res. 58, 806-813.

Wang, W.X., 2012. Biodynamic understanding of mercury accumulation in marine and freshwater fish. Adv. Environ. Res. 1 (1), 15-35.

Wang, W.X., Fisher, N.S., 1999. Delineating metal accumulation pathways for aquatic invertebrates. Sci. Total Environ. 237 (238), 459-472.

Wang, W.X., Wong, R.S.K., 2003. Bioaccumulation kinetics and exposure pathways of inorganic mercury and methylmercury in a marine fish, the sweetlips Plectorhinchus gibbosus. Mar. Ecol-Prog. Ser. 261, 257-268.

Wang, W.X., Griscom, S.B., Fisher, N.S., 1997. Bioavailability of $\mathrm{Cr}(\mathrm{III})$ and $\mathrm{Cr}(\mathrm{VI})$ to marine mussels from solute and particulate pathways. Environ. Sci. Technol. 31 603-611.

Wang, W.X., Stupakoff, I., Gagnon, C., Fisher, N.S., 1998. Bioavailability of inorganic and methylmercury to a marine depositfeeding polychaete. Environ. Sci. Technol. 32, 2564-2571.

Watanabe, C., 2002. Modification of mercury toxicity by selenium: practical importance. Tohoku J. Exp. Med. 196, 71-77.

Whitehead, P.J.P., 1990. Clupeidae. In: Quero, J.C., Hureau, J.C., Karrer, C., Post, A., Saldanha, L. (Eds.), Check-list of the Fishes of the Eastern Tropical Atlantic (CLOFETA), vol. 1, JNICT, Lisbon; SEI, Paris; and UNESCO, Paris. pp. 208-227.

WHO (World Health Organization), 2006. Summary and Conclusions of the Sixty Seventh Meeting of the Joint FAO/WHO Export Committee on Food Additives, Rome, 20-29 June 2006. World Health Organization, Geneva <http:// apps.who.int> (accessed 10.12.12).

Winfrey, M.R., Rudd, J.W.M., 1990. Environmental factors affecting the formation of methylmercury in low pH lakes. Environ. Toxicol. Chem. 9, 853-869.

Wirszubski, A., 1953. On the biology and biotope of the red mullet Mullus barbatus Bull. Sea Fish. Res. Sin, HaOCa (7), 20.

Wolfgang, S., 1983. Mercury content of fishery products: data from the last decade. Sci. Total Environ. 31, 283-300.

$\mathrm{Xu}$, Y., Wang, W.X., 2002. Exposure and food chain transfer factor of Cd, Se, and $\mathrm{Zn}$ in a marine fish, Lutjanus argentimaculatus. Mar. Ecol-Prog. Ser. 238, 173-186. 